ИЗВЕСТИЯ АКАДЕМИИ НАУК ЭСТОНСКОЙ ССР. ФИЗИКА * МАТЕМАТИКА PROCEEDINGS OF THE ACADEMY OF SCIENCES OF THE ESTONIAN SSR. PHYSICS * MATHEMATICS

$1986,35,3$

УДК 621.3.0.16.2.001

Maйpe ОЯВЕЭР, В. САРВ

\title{
О ВЫПОЛНЕНИИ ЗАКОНА СОХРАНЕНИЯ ДЛЯ СОСТАВЛЯЮЩИХ МГНОВЕННОЙ МОЩНОСТИ
}

\author{
(Представил И. Эпик)
}

\section{Введение}

В последнее время заметно повысился интерес к энергетическим методам анализа электрических цепей, применение которых способствует установлению принципиальных возможностей совершенствования разных схем $\left[{ }^{1-34}\right]$. Так как синтезируемая цепь должна обычно обеспечивать требуемые множества токов и напряжений, то при исследовании энергетических процессов удобным является понятие мощности, определяемой произведением тока и напряжения. В зависимости от исследуемой задачи наиболее подходящими могут оказаться те или другие виды мощности, чем, вероятно, и объясняются разные определения ее составляющих.

Наибольшее практическое применение нашло известное понятие полной мощности и ее составляющих в виде активной и реактивной мощностей и мощности искажения $\left[{ }^{1-3}\right]$, подробный обзор которых приведен в $\left[{ }^{1}\right]$, при этом особое внимание обращено на разные определения реактивной мощности. Однако недостатком такого разделения является то, что в цепях при несинусоидальных режимах как для полной мощности, так и для некоторых ее составляющих не выполняется закон сохранения $\left[{ }^{4-6}\right]$. Выражения разных интегральных составляющих мощности выведены из мгновенной мощности, сохранение баланса которой давно известно $\left[{ }^{5-8}\right]$. Выясняется, что из интегральных составляющих мощности, используемых при несинусоидальных режимах, только активная и реактивная обладают свойством сохранения [ $\left.{ }^{6-10}\right]$.

Целью настоящей статьи является выяснить, для каких составляющих временной функции мгновенной мощности в электрической цепи с периодическими напряжениями и токами (в общем случае с несинусоидальными) выполняется закон сохранения.

Это дает возможность получить дополнительные соотношения для анализа и синтеза цепей, а также разделить мгновенную мощность на составляющие, из которых каждая подчиняется закону сохранения.

\section{Различные системы составляющих мгновенной мощности}

Имеется множество исследований, в которых рассматриваются составляющие мгновенной мощности $\left[{ }^{5}, 11-34\right]$.

Разделение мгновенной мощности на постоянную и переменную составляющие давно известно, так как первая из них интегральная активная мощность. По данным [ $\left.{ }^{11}\right]$, уже в первой четверти настоящего столетия нашли применение и понятия мгновенной активной и неактивной 
мощностей. В 1932 f. С. Фризе [ $\left.{ }^{12}\right]$ предложил свою математически й физически подробно обоснованную концепцию о разделении мгновенной мощности двухполюсника с несинусоидальными напряжением и током на мгновенные активную и неактивную составляющие. При этом он показал, что разделение осуществимо двумя способами: 1) исходя из мгновенного напряжения двухполюсника и определяя активную и неактивную составляющие мгновенного тока; 2) исходя из мгновенного тока двухполюсника и определяя соответствующие составляющие мгновенного напряжения.

Следующий шаг в декомпозиции мгновенной мощности сделал в 1933 г. Х. Риссик $\left[{ }^{13}\right]$, разделив мгновенную мощность на три составляющие. Названий он им не дал. Сам он пользовался только названиями активной и реактивной составляющих мгновенной мощности при синусоидальных напряжениях и токах.

Повышение интереса к составляющим мгновенной мощности в 60-е годы $\left[{ }^{14-17}\right]$ и целесообразность использования этих составляющих при анализе преобразовательных процессов $\left[{ }^{24-30}\right]$ сделали необходимым присвоить им определенные названия.

Рассмотрим двухполюсник с несинусоидальными периодическими током $i$ и напряжением $u$. Примем

$$
\begin{aligned}
& u=\sum_{k=0}^{\infty} u_{(k)}=\sum_{k=0}^{\infty} \sqrt{2} U_{(k)} \sin \left(k \omega t+\alpha_{(k)}\right), \\
& i=\sum_{n=0}^{\infty} i_{(n)}=\sum_{n=0}^{\infty} \sqrt{2} I_{(n)} \sin \left(n_{\omega} t+\beta_{(n)}\right) .
\end{aligned}
$$

Каждую гармонику тока можно разделить на активную и реактивную составляющие $\left[{ }^{16,28-30}\right]$

$$
i_{(n)}=i_{(n) p}+i_{(n) q}
$$

Мгновенную мощность $s$ можно представить в виде суммы трех составляющих

$$
\begin{aligned}
s & =u i=\sum_{k=0}^{\infty} u_{(k)} \sum_{n=0}^{\infty} i_{(n)}=\sum_{k=0}^{\infty} u_{(k)} \sum_{n=0}^{\infty}\left(i_{(n) p}+i_{(n) q}\right)= \\
& =\sum_{k=0}^{\infty} u_{(k)} i_{(k) p}+\sum_{k=1}^{\infty} u_{(k)} i_{(k) q}+\sum_{\substack{k, n=0 \\
k \neq n}}^{\infty} u_{(k)} i_{(n)},
\end{aligned}
$$

которые в $\left[{ }^{23,} 28-30\right]$ принято называть мгновенной активной мощностью

$$
p=\sum_{k=0}^{\infty} u_{(k)} i_{(k) p},
$$

мгновенной реактивной мощностью

$$
q=\sum_{k=1}^{\infty} u_{(k)} i_{(k) q}
$$

и мгновенной мощностью искажения

$$
d=\sum_{\substack{k, n=0 \\ k \neq n}}^{\infty} u_{(k)} i_{(n)} .
$$

Название мгновенной мощности искажения впервые предложено нами в $\left[{ }^{27,28}\right]$ по аналогии с соответствующей интегральной величиной. Сумма $(p+q)$ называется мгновенной активно-реактивной мощно- 
стью $\left[{ }^{24-26}\right]$. Мгновенную активную мощность часто подразделяют на̊ постоянную $\left(p_{=}=P\right)$ и переменную $\left(p_{\sim}\right)$ составляющие $[24-26,29]$

$$
p=p_{=}+p_{\sim}=P+p_{\sim} .
$$

Мгновенную мощность можно представить также в виде двух давно известных составляющих - постоянной и переменной

$$
s=p+q+d=p_{=}+p_{\sim}+q+d=s_{2}+s_{\sim}=P+s_{\sim} .
$$

Сумма $(q+d)$ называется мгновенной неактивной (пассивной) мощностью $[25,29,31]$.

Используя вышеприведенные обозначения, разделение мгновенной мощности Х. Риссиком можно представить в виде $s=p+q+d$, а также $s=P+\left(p_{\sim}+q\right)+d$.

Вероятно, целесообразное разделение мгновенной мощности на подходящие составляющие зависит от поставленной задачи.

Так, например, при выборе компенсирующих устройств в $\left[{ }^{29}\right]$ совегуют разделить мгновенную мощность на активную и неактивную составляющие, а в $\left[{ }^{32}\right]$ предлагают новый компенсатор именно мгновенной реактивной мощности.

Отметим, что терминология по составляющим мгновенной мощности не является окончательно установленной. Например, под мгновенной активной мощностью часть авторов понимает сумму мгновенных активных мощностей отдельных гармоник $\left[{ }^{11,13,23,28-30}\right]$, часть - всю мгновенную мощность активного сопротивления $[12,24-26]$. Единство отсутствует и при определении мгновенной реактивной мощности.

В настоящей статье используются определения, соответствующие выражениям (4)-(7), совпадающие с понятиями, приведенными в $[13,23$ $28-30]$.

\section{Составляющие мгновенной мощности, подчиняющиеся закону сохранения}

Рассмотрим электрическую цепь с периодическими несинусоидальными напряжениями и токами, состоящую из $m$ ветвей. Напряжения и токи ветвей

где $l=1, \ldots, m$.

$$
u_{l}=\sum_{k=0}^{\infty} u_{l(k)} ; \quad i_{l}=\sum_{n=0}^{\infty} i_{l(n)}
$$

Мгновенная мощность ветвей выражается

$$
\begin{aligned}
& s_{1}=u_{1} \dot{i}_{1}=\sum_{k=0}^{\infty} u_{1(k)} \sum_{n=0}^{\infty} i_{1(n)}=\sum_{k=0}^{\infty} u_{1(k)} i_{1(k)}+\sum_{\substack{k, n=0 \\
k \neq n}}^{\infty} u_{1(k)} i_{1(n)}= \\
& =\left(p_{1}+q_{1}\right)+d_{1}, \\
& s_{l}=u_{l} i_{l}=\sum_{k=0}^{\infty} u_{l(k)} \sum_{n=0}^{\infty} i_{l(n)}=\sum_{k=0}^{\infty} u_{l(k)} i_{l(k)}+\sum_{\substack{k, n=0 \\
k \neq n}}^{\infty} u_{l(k)} i_{l(n)}= \\
& =\left(p_{l}+q_{l}\right)+d_{l}, \\
& s_{m}=u_{m} i_{m}=\sum_{k=0}^{\infty} u_{m(k)} \sum_{n=0}^{\infty} i_{m(n)}=\sum_{k=0}^{\infty} u_{m(k)} i_{m(k)}+\sum_{\substack{k, n=0 \\
k \neq n}}^{\infty} u_{m(k)} i_{m(n)}= \\
& =\left(p_{m}+q_{m}\right)+d_{m} \text {. }
\end{aligned}
$$


Закон сохранения мгновенной мощности утверждает, что алгебраическая сумма мгновенных мощностей всех элементов цепи равняется нулю. Следовательно,

$$
s=\sum_{l=1}^{m} s_{l}=\sum_{l=1}^{m} u_{l} i_{l}=\sum_{l=1}^{m}\left(p_{l}+q_{l}\right)+d_{l}=0 .
$$

Известно, что законы Кирхгофа действительны отдельно для постоянной составляющей $(k, n=0)$ и для каждой гармоники тока и напряжения $\left[{ }^{10}\right]$. Поэтому на основе теоремы Телледжена $\left[{ }^{7,35}\right]$ произведения любой пары гармоник напряжения и тока, суммированные через все ветви, равны нулю. Следовательно,

$$
\begin{aligned}
& \sum_{l=1}^{m} u_{l(0)} i_{l(0)}=0, \sum_{l=1}^{m} u_{l(0)} i_{l(1)}=0, \ldots, \sum_{l=1}^{m} u_{l(0)} i_{l(j)}=0, \ldots \\
& \sum_{l=1}^{m} u_{l(1)} i_{l(0)}=0, \sum_{l=1}^{m} u_{l(1)} i_{l(1)}=0, \ldots, \sum_{l=1}^{m} u_{l(1)} i_{l(j)}=0, \ldots \\
& . . . . . . . . . . . . . \\
& \sum_{l=1}^{m} u_{l(j)} i_{l(0)}=0, \sum_{l=1}^{m} u_{l(j)} i_{l(1)}=0, \ldots, \sum_{l=1}^{m} u_{l(j)} i_{l(j)}=0, \ldots
\end{aligned}
$$

Используя понятия составляющих мгновенной мощности, приведенные в формулах (5)-(7), уравнения (13) приобретают вид:

$$
\begin{aligned}
& \sum_{l=1}^{m} p_{l(0)}=0, \sum_{l=1}^{m} d_{l(0,1)}=0, \ldots, \sum_{l=1}^{m} d_{l(0, j)}=0, \ldots \\
& \sum_{l=1}^{m} d_{l(1,0)}=0, \sum_{l=1}^{m}\left(p_{l(1)}+q_{l(1)}\right)=0, \ldots, \sum_{l=1}^{m} d_{l(1, j)}=0, \ldots \\
& \sum_{l=1}^{m} d_{l(j, 0)}=0, \sum_{l=1}^{m} d_{l(j, 1)}=0, \ldots, \sum_{l=1}^{m}\left(p_{l(j)}+q_{l(j)}\right)=0 ; \ldots
\end{aligned}
$$

Суммируя все элементарные составляюшше мгновенной мощности, приведенные в (14), получаем закон сохранения мгновенной мощности

$$
\sum_{l=1}^{m} \sum_{k=0}^{\infty} u_{l(k)} \sum_{n=0}^{\infty} i_{l(n)}=\sum_{l=1}^{m} s_{l}=0
$$

На основе (13) и (14) можно написать также

$$
\begin{aligned}
& \sum_{l=1}^{m} \sum_{k=0}^{\infty} u_{l(k)} i_{l(k)}=\sum_{l=1}^{m} \sum_{k=0}^{\infty}\left(p_{l(k)}+q_{l(k)}\right)=\sum_{l=1}^{m}\left(p_{l}+q_{l}\right)=0, \\
& \sum_{l=1}^{m} \sum_{\substack{k, n=0 \\
k \neq n}}^{\infty} u_{l(k)} i_{l(n)}=\sum_{l=1}^{m} \sum_{\substack{k, n=0 \\
k \neq n}}^{\infty} d_{l(k, n)}=\sum_{l=1}^{m} d_{l}=0 .
\end{aligned}
$$

Это означает, что баланс сохраняется как для мгновенной активнореактивной мощности, так и для мгновенной мощности искажения. Разделяя по (8) $\quad p_{l}=P_{l}+p_{l \sim}$, получаем 


$$
\sum_{l=1}^{m}\left(p_{l}+q_{l}\right)=\sum_{l=1}^{m}\left(P_{l}+p_{l \sim}+q_{l}\right)=\sum_{l=1}^{m} P_{l}+\sum_{l=1}^{m}\left(p_{l \sim}+q_{l}\right) .
$$

Формулы (12) и (18) дают

$$
s=\sum_{l=1}^{m} s_{l}=\sum_{l=1}^{m} P_{l}+\sum_{l=1}^{m}\left(p_{l \sim}+q_{l}\right)+\sum_{l=1}^{m} d_{l}=0 .
$$

Так как $\sum_{l=1}^{m} P_{l}=0$, то и $\sum_{l=1}^{m}\left(p_{l \sim}+q_{l}\right)=0$.

Из вышеизложенного вытекает, что закон сохранения выполняется для постоянной составляющей мгновенной мощности, для переменной составляющей мгновенной активно-реактивной мощности и для мгновенной мощности искажения. Для доказательства этого мы использовали теорему Телледжена, которая показывает, что законы Кирхгофа являются достаточными, чтобы доказать сохранение энергии и мощности в электрической цепи, несмотря на свойства ее элементов.

Существенно подчеркнуть, что мгновенная активно-реактивная мощность какой-то гармоники является мгновенной мощностью этой гармоники, причем закон сохранения выполняется отдельно для постоянной и переменной составляющих мгновенной мощности любой гармоники. Важно и то, что мгновенная активно-реактивная мощность любой гармоники (а следовательно, и всех гармоник в целом) не зависит от того, определены ли мгновенные активная и реактивная мощности на основе разделения на активную и реактивную составляющие тока или напряжения.

В целях иллюстрации выполнения закона сохранения для составляющих мгновенной мощности приведем несколько примеров. Эти же примеры одновременно показывают, что в общем случае закон сохранения не выполняется отдельно для мгновенных активной и реактивной мощностей.

Пример 1. Рассматриваемая схема приведена на рис. 1. На рис 2. показаны временные диаграммы напряжений, токов и мощностей в схеме при $\quad u=U_{m} \sin \omega t ; U_{m}=1, R_{1}=R_{2}=1, x_{2}=\omega L_{2}=1, z_{2}=\sqrt{R_{2}^{2}+x_{2}^{2}}=$ $=\sqrt{2}, \varphi_{2}=\pi / 4$. Как видно из рис. 2 , баланс выполняется в каждый момент времени только для мгновенной активно-реактивной мощности $p^{\prime}+q^{\prime}=p^{\prime \prime}+q^{\prime \prime}=p_{1}+p_{2}+q_{2}$ и для ее постоянной и переменной составляющих. Если разделить $p^{\prime}=P+p_{\sim}^{\prime}$ и $p_{1}+p_{2}+q_{2}=P_{1}+P_{2}+p_{1 \sim}+$ $+p_{2 \sim}+q_{2}$, то $P=P_{1}+P_{2}$ и $p_{\sim}^{\prime}+q^{\prime}=p_{1 \sim}+p_{2 \sim}+q_{2}$, но $p^{\prime} \neq p_{1}+p_{2} \neq p^{\prime \prime}$ и $q^{\prime} \neq q_{2} \neq q^{\prime \prime}$, т. е. баланс не выполняется для мгновенных активной и реактивной мощностей отдельно.

Математически это связано с тем, что в общем случае активные и реактивные составляющие мгновенных токов и/или напряжений не подчиняются законам Кирхгофа, что видно уже из простой схемы (рис. 1). Так, в контуре $R_{1}, R_{2}, L_{2}$ второй закон Кирхгофа не выполня-

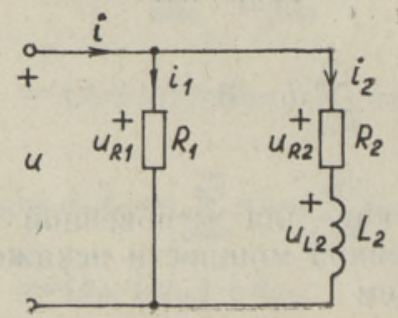

Рнс. 1. 

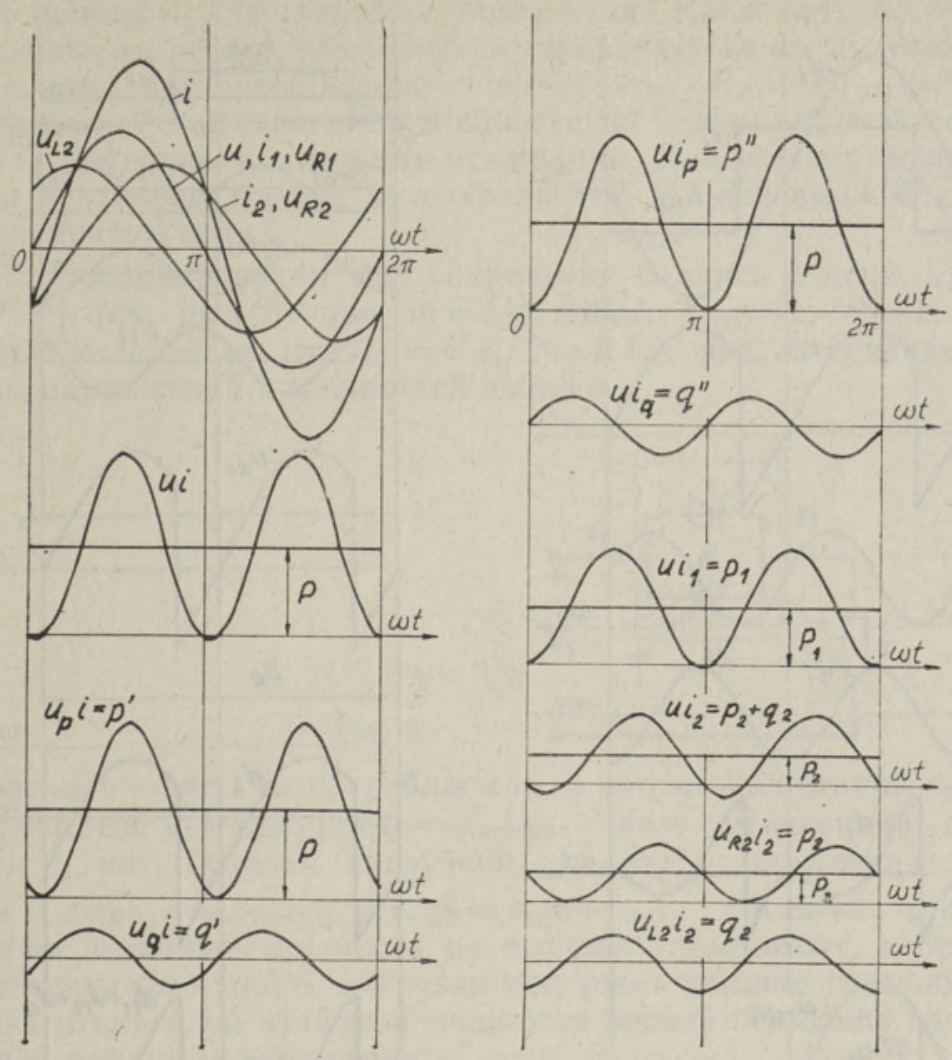

Рис. 2.

ется ни для активных, ни для реактивных составляющих мгновенных напряжений, т. е. $u_{R 1}-u_{R 2} \neq 0$ и $u_{L 2}+0 \neq 0$.

C физической точки зрения невыполнение закона сохранения для активной и реактивной составляющих мгновенной мощности объясняется тем, что в общем случае энергия, генерированная реактивными элементами, не возвращается в источник питания непосредственно.

Пример. 2. Рассмотрим обмен энергии и баланс мгновенных мощностей в простой идеализированной цепи на рис. 3, содержащей ключевые элементы. Допустим $u_{1}=U_{1 m} \sin \omega t, L=\infty, U_{1 m}=1, R=1, \alpha=\pi / 4$, $U_{R L(0)}=I_{0}$. Временные диаграммы напряжений, токов и мощностей всех элементов схемы приведены на рис. 4, где $u_{1}, i_{1}-$ напряжение и ток питания; $s=u_{1} i_{1}-$ мощность, потребляемая из источника питания; $p_{1}=u_{1 p} i_{1(1)} ; \quad q_{1}=u_{1 q} i_{1(1)} ; \quad p_{T 1(1)}=u_{T 1(1)} i_{T 1(1)} ; \quad q_{T 1(1)}=u_{T 1(1) q} i_{T 1(1)} ; \quad p_{T 1(0)}=$ $=u_{T 1(0)} i_{T 1(0)}$.

Рис. 3.

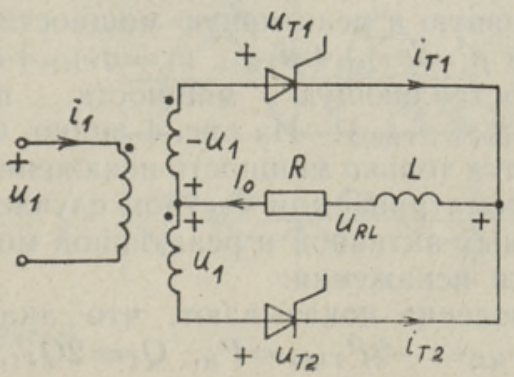



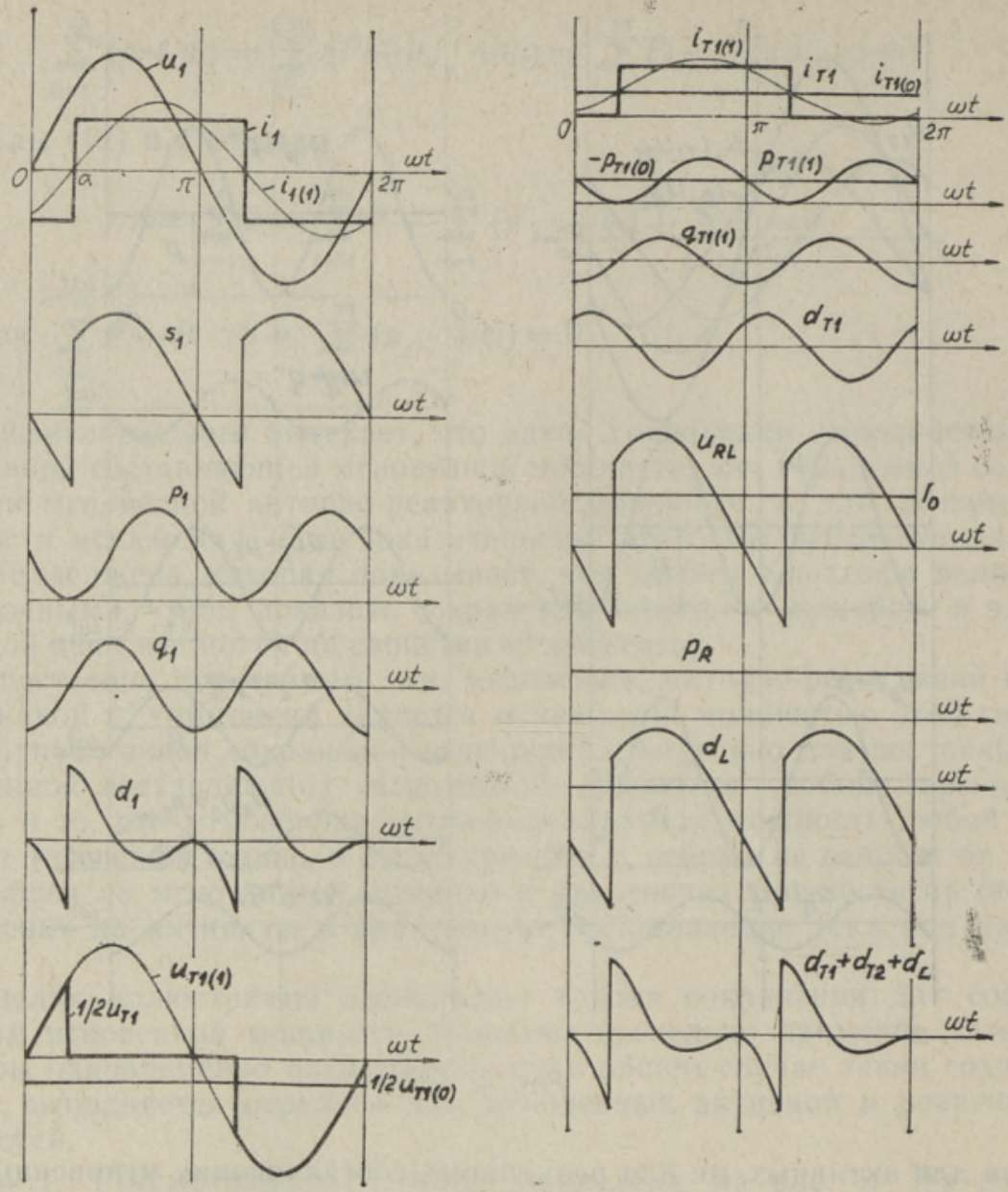

Рис. 4.

Так как напряжение ключей $\quad u_{T 1}=u_{T 1(0)}+u_{T 1(1)}+\sum_{n=1}^{\infty} u_{T 1(2 n)}, \quad$ а ток ключей $\quad i_{T 1}=i_{T 1(\theta)}+i_{T 1(1)}+\sum_{n=1}^{\infty} i_{T 1(2 n+1)}$, то $p_{T 1}=p_{T 1(1)}+p_{T 1(0)}, \quad q_{T 1}=q_{T 1(1)}$.

Кривые входной мощности $s_{1}=p_{1}+q_{1}+d_{1}$ и мощности цепи нагрузки $s_{R L}=p_{R}+d_{L}$ совпадают. Мгновенная мощность ключа всегда равняется нулю $\left(s_{T 1}=p_{T 1}+q_{T 1}+d_{T 1}=0\right)$. Источник питания генерирует мгновенные активную и реактивную мощности первой гармоники, потребляемые ключами $p_{1}=p_{T 1(1)}+p_{T 2(1)}, q_{1}=q_{T 1(1)}+q_{T_{2}(1)}$. Ключи генерируют постоянную составляющую мощности, потребляемую нагрузкой $p_{R}=$ $=-\left(p_{T 1(0)}+p_{T 2(0)}\right)$. Из рис. 4 видно, что $d_{1}=d_{T 1}+d_{T 2}+d_{L}$. На дросселе выделяется только мощность искажения $d_{L}=u_{L} I_{0}$.

В рассматриваемом частном случае баланс сохраняется отдельно для мгновенных активной и реактивной мощностей, а также для мгновенной мощности искажения.

Вычисления показывают, что аналогично мгновенным мощностям $P_{1}=2 P_{T 1(1)}=-2 P_{T 1(0)}=P_{R}, Q_{1}=2 Q_{T 1}, S_{1}=S_{R L}$. 
Активная мощность ключа всегда равна нулю. Ключ потребляет активную мощность на первой гармонике и генерирует ее на нулевой гармонике, т. е. работает преобразователем частоты: $P_{T 1}=P_{T 1(1)}+P_{T 1(0)}=0$. Из интегральных составляющих мощности на ключе выделяется только реактивная мощность и мощность искажения. Для полных мощностей и мощности искажения баланс не сохраняется. Их невозможно суммировать по элементам цепи.

Пример 3. Проанализируем еще сохранение баланса в цепи, приведенной в [22,34] (см. рис. 5) при $u_{1}=U_{1 m} \sin \omega t, U_{1 m}=1, R=1, \alpha=\pi / 4$. При $\alpha=0 i_{1}=I_{1 m} \sin \omega t$, где $I_{1 m}=U_{1 m} / R=1$. На рис. 6 приведены кривые токов, напряжений и мощностей в схеме.

Рис. 5.

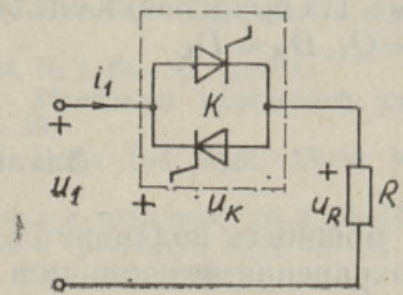

Мгновенная мощность, потребляемая из источника питания, $s_{1}=u_{1} i_{1}=$ $=p_{1}+q_{1}+d_{1}$, где $p_{1}=u_{1 p} i_{1(1)}$ и $q_{1}=u_{1 q} i_{1(1)}$. Часть мгновенной активной мощности $p_{1}$ потребляется нагрузкой $p_{R(1)}=i_{1(1)}^{2}$, остальная часть ключами $p_{K(1)}=u_{K(1) p} i_{1(1)}$, т. е. $p_{1}=p_{R(1)}+p_{K(1)}$. Ключи генерируют мгновенную активную мощность на высших гармониках, в результате чего мгновенная мощность нагрузки содержит высшие гармоники. Она состоит из мгновенной активной мощности первой и высших гармоник и мгновенной мощности искажения

$$
S_{R}=p_{R(1)}+\sum_{n=2}^{\infty} p_{R(n)}+d_{R}
$$
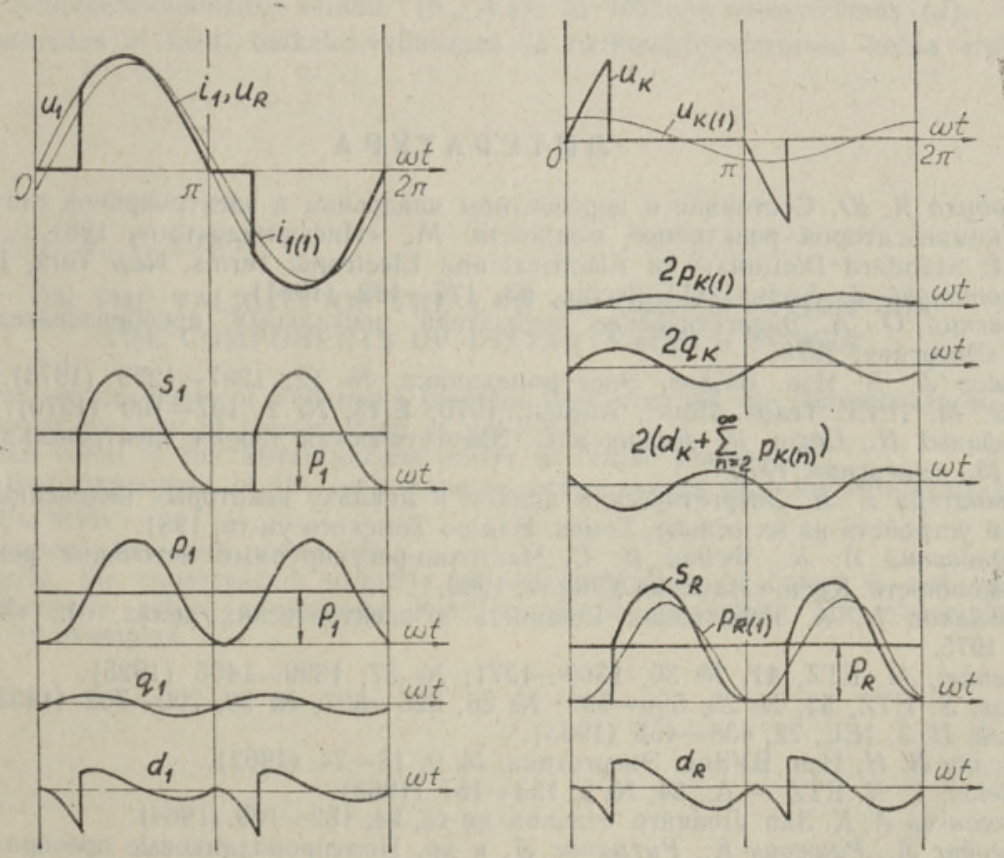

Рис. 6. 
Йз рис. 6 видно, что $s_{1}=s_{R}$. Как известно, $s_{K}=0$. (На рисунке все кривые мощностей даны в уменьшенном масштабе (1:2) по сравнению с кривыми тока и напряжения.) Так как кривые реактивных составляющих первой гармоники напряжения ключей и питания совпадают, то $q_{K(1)}=u_{1 q} i_{1(1)}=q_{1}$. О мгновенных мощностях искажения можно сказать, что $d_{1}=d_{R}+d_{K}$.

В этом частном случае баланс выполняется отдельно для мгновенных активной и реактивной мощностей и мощности искажения. Но если для нахождения кривых мощностей использовать активную и реактивную составляющие первой гармоники тока, т. е. $i_{1(1)}=i_{1(1) p}+i_{1(1) q}$ и соответственно $p_{1}=u_{1} i_{1(1) p} \quad$ и $\quad q_{1}=u_{1} i_{1(1) q} ; \quad p_{K(1)}=u_{K(1)} i_{1(1) p} \quad$ и $\quad q_{K(1)}=$ $=u_{K(1)} t_{1(1) q}$, то баланс отдельно для них не выполняется даже в этой простой схеме. Из вычислений следует, что $S_{R}=P_{R}=P_{1}, Q_{R}=0, D_{R}=0$, $P_{K}=0, Q_{K}=Q_{1}, D_{K}=D_{1}$.

\section{Заключение}

Мгновенная мощность подчиняется закону сохранения. Из ее компонентов закон сохранения выполняется для постоянной составляющей, переменной составляющей мгновенной активно-реактивной мощности и для мгновенной мощности искажения.

Для мгновенных активной и реактивной мощностей отдельно в общем случае закон сохранения не выполняется.

Дополнительные соотношения, выражающие закон сохранения для отдельных определенных составляющих временной функции мгновенной мощности, целесообразно применять при уточнении преобразовательных процессов в цепях с периодическими несинусоидальными токами и напряжениями, например, в цепях ключевых преобразователей параметров электроэнергии.

\section{ЛИТЕРА Т У РА}

1. Солодухо Я. Ю. Состояние и перспективы внедрения в электропривод статических компенсаторов реактивной мощности. М., «Информэлектро», 1981.

2. IEEE Standard Dictionary of Electrical and Electronic Terms. New York, 1978.

3. Novomiejski, Z. Arch. Elektrotechn., 63, 177-182 (1981).

4. Маевский O. А. Энергетнческие показатели вентильных преобразователей. М., «Энергия», 1978.

5. Бычков Л. В. Изв. ВУЗов. Электромеханика, № 12, 1287-1293 (1973).

6. Milič, M. IEEE Trans. Educ., August, 1970, E-13, № 2, 107-109 (1970).

7. Пенфильд П., Спенс Р., Дюинкер С. Энергетическая теория электрических цепей. М., «Энергия», 1970.

8. Гольдитейн Е. Н. Энергетический подход к анализу некоторых нелинейных цепей и устройств на их основе. Томск, Изд-во Томского ун-та, 1981.

9. Шидловский $A$. K., Федий $B$. C. Частотно-регулируемые источники реактнвной мощности. Киев, «Наукова думка», 1980.

10. Мельников H. A. Реактивная мощность в электрических цепях. М., «Энергия», 1975.

11. Schenkel, M. ETZ, 47, № 36, 1369-1371; № 37, 1399-1405 (1925).

12. Fryze, S. ETZ, 53, № 25, 596-599; № 26, 625-627; № 29, 700-702 (1932).

13. Rissik, H. J. IÉE, 72, 435-455 (1933).

14. Неделку В. Н. Изв. ВУЗов. Энергетика, № 9, 18-24 (1962).

15. Nedelcu, V. N. ETZ - A., 84, № 5, 153-157 (1963).

16. Дарманчев А. К. Зап. Ленингр. сельхоз. ин-та, 94, 182-189 (1964).

17. Крогерис А., Рашевич К., Рутманис Л. и др. Полупроводниковые преобразователи электрической энергии. Рига, «Зинатне», 1969.

18. Rosman, H. Bul. Inst. politechn. Iasi, XIX (XXIII), 3-4, 9-16 (1973). 
19. Сидорович А. М. Электричество, № 11, 12-16 (1979).

20. Tsuboi Kunio, Harashima Fumio. IECI Ann. Conf. Proc. Appl. Microprocess, Philadelphia, Pa., 1977. New York, 1977, 415-418.

21. Sheperd, W. Energy Flow and Power Factor in Nonsinusoidal Circuits. Cambridge, University Press, 1979.

22. Милях А. Н., Жарский $Б$. К. В кн.: Проблемы технической электродинамики, вып. 62. Киев, «Наукова думка», 1977, 3-7.

23. Emanuel, A. E. Árch. Elektrotechn., 59, 183-189 (1977).

24. Зиновьев Г. С. В кн.: Тиристорные преобразователи частоты. Новосибирск, Новосиб. электротехн. нн-т, 1981, 3-28.

25. Зиновьев Г. С. В кн.: Снловая полупроводниковая техника. Новосибирск, Новосиб. электротехн. ин-т, 1982, 133-141.

26. Зиновьев Г. С. Препринт-342 ИЭД АН УССР. Киев, 1983.

27. Олвеэр М., Сарв В. Изв. АН ЭССР. Фнз. Матем., 24, № 1, 78-81 (1975).

28. Оявеэр М., Сарв В. Изв. АН ЭССР. Физ. Матем., 24, № 4, 442-443 (1975).

29. Крогерис А. Ф., Трейманис Э. П. Изв. АН ЛатвССР. Сер. физ. и техн. наук, № 6, $22-31(1984)$.

30. Оявеэр М. Изв. АН ЭССР. Физ. Матем., 34, № 1, 86-92 (1985).

31. Вuселов Д. В., Гальперин В. E. В кн.: Проблемы нелинейной электротехннки, ч. 1. Киев, «Наукова думка», 1984. 38-40.

32. Akagi, H., Kanazawa, Y., Nabae, A. IEEE Trans. Ind. Appl., IA-20. № 3, 625-630 (1984)

33. Демирчян К. С. Изв. АН СССР. Энергетика и транспорт, № 2, 66-72 (1984).

34. Жарков Ф. П. Изв. АН СССР. Энергетика и транспорт, № 2, 73-81 (1984).

35. Tellegen, B. D. H. Philips Res. Repts, 7, 259-269 (1952).
Институт термофизики и электрофизики Академии наук Эстонской ССР
Поступила в редакцию $10 / \mathrm{X} 1985$

Maire OJAVEER, V. SARV

\section{HETKVOIMSUSE KOMPONENTIDE ALLUVUSEST JAÄVUSSEADUSELE}

Kasutades Tellegeni teoreemi on tõestatud, et jäävusseadusele alluvad järgmised hetkvõimsuse komponendid: 1) alaliskomponent $(P) ; 2$ ) hetkaktiivvõimsuse vahelduvkomponendi ja hetkreaktiivvõimsuse summa $\left(p_{\sim}+q\right)$; 3$)$ hetkmoonutusvōimsus $(d)$.

Jäävusseadus ei kehti hetkaktiivvõimsuse ja hetkreaktiivvõimsuse kohta eraldi.

Maire OJAVEER, V. SARV

\section{ON THE VALIDITY OF THE LAW OF CONSERVATION FOR THE COMPONENTS OF INSTANTANEOUS POWER}

It is proved on the basis of Tellegen's theorem that from all the components of instantaneous power the law of conservation is valid for

1) the mean value of the instantaneous power or active power $(P)$,

2) alternating component of the instantaneous active power plus instantaneous reactive power $(p \sim q)$,

3) instantaneous distortion power $(d)$.

Generally, the conservation property of the instantaneous power is not valid for the instantaneous active power and reactive power separately. The above-stated is illustrated by examples. 